\title{
Alcohol Consumption More than 2 Drinks per Day for Men and More than 1 Drink per Day for Women
}

National Cancer Institute

\section{Source}

National Cancer Institute. Alcohol Consumption More than 2 Drinks per Day for Men and More than 1 Drink per Day for Women. NCI Thesaurus. Code C156821.

Alcohol consumption more than two drinks per day for men and more than one drink per day for women. 